\title{
Spatial Organization of Seven Extreme Solar Energetic Particle Events
}

\author{
Leon Kocharov $^{1}{ }^{1}$, Silja Pohjolainen ${ }^{2}$, Mike J. Reiner $^{3,4}$, Alexander Mishev ${ }^{5}$, \\ Haimin Wang ${ }^{6,7}$ (1) , Ilya Usoskin ${ }^{1,5}$ (10, and Rami Vainio ${ }^{8}$ \\ ${ }^{1}$ Sodankylä Geophysical Observatory, University of Oulu, P.O.B. 3000, Oulu FI-90014, Finland \\ ${ }^{2}$ Tuorla Observatory, University of Turku, Piikkiö FI-21500, Finland \\ ${ }^{3}$ The Catholic University of America, Washington, DC, USA \\ ${ }^{4}$ NASA/Goddard Space Flight Center, Greenbelt, MD, USA \\ ${ }^{5}$ Space Climate Research Unit, University of Oulu, Oulu FI-90014, Finland \\ ${ }^{6}$ Space Weather Research Laboratory, New Jersey Institute of Technology, Newark, NJ 07102-1982, USA \\ ${ }^{7}$ Big Bear Solar Observatory, New Jersey Institute of Technology, Big Bear City, CA 92314, USA \\ ${ }^{8}$ Space Research Laboratory, University of Turku, Turku FI-20014, Finland \\ Received 2018 January 12; revised 2018 June 29; accepted 2018 July 4; published 2018 July 31
}

\begin{abstract}
Emission of relativistic protons and helium responsible for extreme solar particle events (ground level enhancements (GLEs)) is often structured. We investigate its organization depending on the eruption stage characterized by the heliocentric height of associated coronal mass ejections (CMEs). Seven GLEs are considered: events on 1997 November 6, 1998 May 2, 2000 July 14, 2001 December 26, 2003 November 2, 2006 December 13, and 2012 May 17, which are half of the SOlar and Heliospheric Observatory (SOHO)-era GLEs, excluding very weak events. Count-rate profiles of the GLEs plotted as a function of the CME height reveal two types (or two components) of the high-energy particle emission. The first component rises in a steplike manner during the $\mathrm{CME}$ transit from $2 R_{\odot}$ to $3 R_{\odot}$, when the CME exits from predominantly closed coronal magnetic structures, irrespective of the CME speed (type H). This component is of coronal origin. The second component of the GLE-producing particles starts to rise when CME is at about $4 R_{\odot}$, achieves its maximum at 6-10 $R_{\odot}$, and declines shortly after that (type $\mathrm{J}$ ). The type $\mathrm{J}$ particle injection into the interplanetary space coincides with the decametric-hectometric radio burst complex that includes enhanced emission of type II and concurrent low-frequency type III bursts, indicative of the CME interaction with a streamer-like structure at a few solar radii from the Sun. Those could be delayed particles from the flare region. A possible additional contribution of the CME-bow-shock acceleration in unstructured solar wind is not large in the two considered types of events.
\end{abstract}

Key words: acceleration of particles - shock waves - Sun: coronal mass ejections (CMEs) - Sun: flares - Sun: particle emission - Sun: radio radiation

\section{Introduction}

In extreme solar energetic particle (SEP) events, protons can be accelerated to relativistic energies, so that they trigger a nuclear cascade in the atmosphere of the Earth and by this expedient can cause significant signal even in ground-based detectors like neutron monitors (NMs); this is known as a ground level enhancement (GLE) event (Shea \& Smart 2012, and references therein). Possible origins of GLE particles are often discussed in terms of the impulsive-gradual classification of SEP events (flare-accelerated helium-rich versus coronal mass ejection (CME)-accelerated helium-poor; e.g., Reames 1999; Cliver 2016), yet hybrid scenarios cannot be ruled out.

In an attempt to reveal the possible sources of GLEs, it is a common practice to estimate the timing of the first solar protons observed above the galactic background and to compare it with data of solar electromagnetic observations (e.g., Aschwanden 2012). However, the proton fluxes often continue to rise until the $\mathrm{CME}$ heights reach 5-15 $R_{\odot}$ (Kahler 1994). The "first particle" consideration seems insufficient, because emission of GLE-producing protons at/near the Sun may be both prolonged and structured (e.g., Kocharov et al. 2017).

In this Letter, we consider the profiles of seven wellobserved GLEs - the events on 1997 November 6, 1998 May 2, 2000 July 14, 2001 December 26, 2003 November 2, 2006
December 13, and 2012 May 17-as functions of CME heights. Additionally discussed are a historic GLE of 1990 May 24 and the latest GLE observed on 2017 September 10. Our goal is to reveal and classify different structures of the GLE profiles and to relate those structures to different stages of solar eruption development.

\section{CME Kinematics and GLE Profiles}

We analyze events listed in Table 1 using the GLE database stored in Oulu (http://gle.oulu.fi) and the CME height-time data from the Large Angle and Spectrometric COronagraph (LASCO) instrument on the SOlar and Heliospheric Observatory spacecraft (SOHO; Brueckner et al. 1995). Due to the LASCO field of view and cadence limitations, the data are available only for CMEs in the outer corona, at $R>3 R_{\odot}$. As the first emission of GLEproducing particles often occurs when a CME is below $3 R_{\odot}$, some model should be adopted to extrapolate the LASCOobserved height-time profiles back to the Sun (like the three models by Gopalswamy et al. 2012). Here we introduce an exponential self-similar model of a CME launch, which is calibrated with $\mathrm{CME}$ images in the low corona available from other instruments in two GLE-productive eruptions-2003 November 2 and 2012 May 17 (Gopalswamy et al. 2013; Kocharov et al. 2017). 
Table 1

Summary of Considered Flare-CME-GLE Events

\begin{tabular}{|c|c|c|c|c|c|c|c|c|}
\hline $\begin{array}{l}\text { GLE } \\
\text { No. }\end{array}$ & $\begin{array}{l}\text { Event } \\
\text { Date }\end{array}$ & $\begin{array}{c}\text { Flare } \\
\text { Impulsive Phase } \\
(\mathrm{UT})^{\mathrm{a}}\end{array}$ & $\begin{array}{c}\text { Flare } \\
\text { Location } \\
(\mathrm{H} \alpha)\end{array}$ & $\begin{array}{c}\text { Flare } \\
\text { Class } \\
\text { (X-rays) }\end{array}$ & $\begin{array}{c}\text { CME } \\
\text { Speed }^{\mathrm{b}} \\
\left(\mathrm{km} \mathrm{s}^{-1}\right)\end{array}$ & $\begin{array}{c}\text { GLE } \\
\text { Amplitude }^{c} \\
(\%)\end{array}$ & $\begin{array}{c}\text { GLE } \\
\text { Width }^{\mathrm{d}} \\
\left(^{\circ}\right)\end{array}$ & $\begin{array}{c}\text { GLE } \\
\text { Morphological }_{\text {Type }^{\mathrm{e}}}\end{array}$ \\
\hline 55 & 1997 Nov 06 & $11: 52-11: 56$ & S18 W63 & X9.4 & 1556 & 11 & $65^{\mathrm{f}}$ & $\mathrm{J}+$ \\
\hline 59 & $2000 \mathrm{Jul} 14$ & $10: 13-10: 24$ & N22 W07 & X5.7 & 1674 & 40 & $100^{\mathrm{h}}$ & $\mathrm{H}$ \\
\hline 63 & 2001 Dec 26 & $05: 02-05: 20$ & N08 W54 & M7.1 & 1446 & 8 & $\sim 40^{\mathrm{i}}$ & $\mathrm{J}$ \\
\hline 67 & 2003 Nov 02 & $17: 14-17: 18$ & S14 W56 & $\mathrm{X} 8.3$ & 2598 & 15 & $90^{\mathrm{j}}$ & $\mathrm{H}+\mathrm{J}$ \\
\hline
\end{tabular}

Notes.

${ }^{a}$ In hard X-rays or, if the X-ray data are not available, in microwaves.

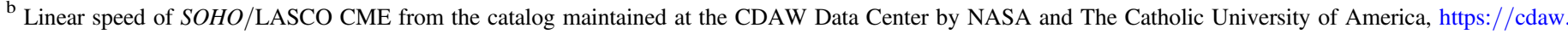
gsfc.nasa.gov (Yashiro et al. 2004).

c At stations shown in Figures 2 and 3.

${ }^{d}$ Pitch angle distribution width at half height.

e Morphological types $\mathrm{H}$ and $\mathrm{J}$ are illustrated with Figure 4; type $\mathrm{J}+$ stands for type $\mathrm{J}$ followed by an additional emission after $\mathrm{CME}$ has reached $10 R_{\odot}$.

${ }^{\mathrm{f}}$ Lovell et al. (2002).

For $20 \mathrm{MeV}$ protons (Kocharov et al. 2007).

h Vashenyuk et al. (2003).

${ }^{\mathrm{i}}$ Present estimate.

${ }^{j}$ Kocharov et al. (2017).

${ }^{\mathrm{k}}$ Vashenyuk et al. (2008).

${ }^{1}$ Mishev et al. (2014).

We propose that during the $\mathrm{CME}$ launch period, at $R \lesssim 10 R_{\odot}$, CME acceleration declines exponentially in time:

$$
\ddot{R}=a \exp \left[-\left(t-t_{\mathrm{C}}\right) / \tau\right],
$$

where $a$ is the maximum acceleration value, $\tau$ is the acceleration timescale, and the CME launch time $t_{\mathrm{C}}=t_{\mathrm{F}}+\delta_{\mathrm{C}}$ with $t_{\mathrm{F}}$ is the start time of the flare impulsive phase and $\delta_{\mathrm{C}}$ is the CME offset time that in the eruptions considered here will be typically but not necessarily positive. The straightforward integration of Equation (1) yields the CME height-time profile

$$
\begin{aligned}
R(t)= & R_{\mathrm{C}}+a \tau^{2}\left[\exp \left(-\frac{t-t_{\mathrm{F}}-\delta_{\mathrm{C}}}{\tau}\right)-1\right] \\
& +a \tau\left(t-t_{\mathrm{F}}-\delta_{\mathrm{C}}\right),
\end{aligned}
$$

where $R_{\mathrm{C}}$ is the CME launch height. The asymptotic speed value is $V_{a}=a \tau$ ( $V_{a}$ would be an intermediate asymptote, if a late deceleration phase was also included). A set of self-similar profiles of faster or slower CMEs, with asymptotic speeds $V_{a}=\rho \times a \tau \equiv \rho \times V_{a o}$, may be obtained by re-scaling the time $t-t_{\mathrm{F}}$ in Equation (1) with the factor $\rho$. That yields in terms of the re-scaled time

$$
\begin{aligned}
R(t)= & R_{\mathrm{C}}+V_{a o} \tau\left[\exp \left(-\frac{t-t_{\mathrm{F}}-\delta_{\mathrm{C}} / \rho}{\tau / \rho}\right)-1\right] \\
& +\rho V_{a o}\left(t-t_{\mathrm{F}}-\delta_{\mathrm{C}} / \rho\right),
\end{aligned}
$$

where $V_{a o}$ is a normalization speed adopted hereafter as $1500 \mathrm{~km} \mathrm{~s}^{-1}$. This set of profiles is used to fit and extrapolate the CME height-time data.

The dotted curve in the upper panel of Figure 1 shows the CME profile with the asymptotic speed $V_{a}=V_{a o} \equiv 1500 \mathrm{~km} \mathrm{~s}^{-1}$, the decay time $\tau=6.74$ minutes, the launch height $R_{\mathrm{C}}=1.6 R_{\odot}$, and the offset time $\delta_{\mathrm{C}}=5$ minutes (Model 1). The corresponding initial acceleration value $a=3.71 \mathrm{~km} \mathrm{~s}^{-2}$. These parameters were adjusted to fit at $\rho=1.75$ the height-time profile observed in the low corona in the 2003 November 2 event (Figure 1). The model acceleration value for that event will be $a=3.71 \rho^{2} \mathrm{~km} \mathrm{~s}^{-2}=$ $11.4 \mathrm{~km} \mathrm{~s}^{-2}$.

The upper panel of Figure 1 shows that by varying only one parameter, $\rho$, it is possible to fit the data of five events. However, in two other events, the CME data require zero or even negative offset time $\delta_{\mathrm{C}}$ (the lower panel of Figure 1, Models 2 and 3).

Comprehensive analysis of a GLE event should comprise the modeling of the particle source near the Sun and the particle transport in interplanetary space and Earth's magnetosphere, followed by the production of a nuclear cascade in the Earth's atmosphere and the detection of secondaries by the NM network (e.g., Kocharov et al. 2017). The modeling indicates, however, that the rise and maximum phase of a selected NM time profile after shifting back in time can indicate the solar source operational period, if two conditions are met: (i) the GLE is sufficiently anisotropic (that is, the pitch angle distribution width in the GLE maximum phase $\lesssim 90^{\circ}$ at half height), and (ii) the NM viewing direction outside of the magnetosphere is close to the particle flux axis (Kocharov et al. 2015, 2017, Figure 1 and the last panel of Figure 8 of those two papers, respectively).

The time-shift value depends on interplanetary magnetic field (IMF) line length and scattering conditions. While the IMF may deviate from the standard field of Parker's solar wind model, e.g., due to a previous CME, we do not find signatures of significant IMF line elongation in the velocity dispersion of deka-MeV protons (Kocharov et al. 2007). The effect of interplanetary scattering is estimated by the modeling of proton transport in the standard IMF and comparison with observed pitch angle distribution, which results in the transport time $\Delta t=11-14$ minute, depending on the event as indicated in Figures 2 and $3, \pm 1$ minute. 


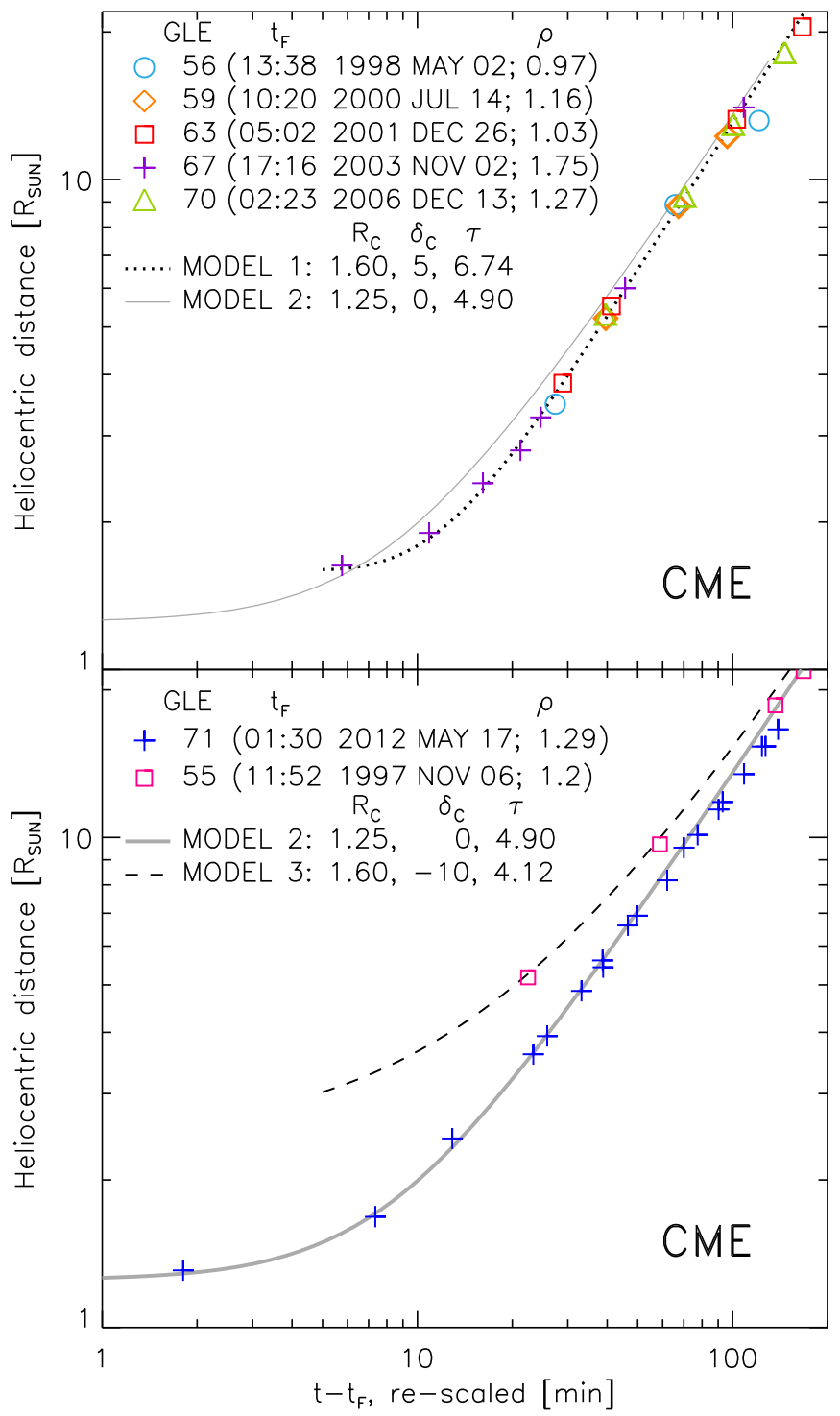

Figure 1. Height-time profiles of seven GLE-associated CMEs (points) and analytical profiles from the exponential acceleration model with the asymptotic speed $V_{a}=V_{a o} \equiv 1500 \mathrm{~km} \mathrm{~s}^{-1}$ (curves). The timing of each CME has been rescaled with the factor $\rho$ indicated in the figure, $\left(t-t_{\mathrm{F}}\right)_{\text {re-scaled }}=\left(t-t_{\mathrm{F}}\right) \rho$, so that in terms of the re-scaled time, all of the CMEs are of the same asymptotic speed $V_{a o}$. The low corona data are available and employed for GLEs 67 and 71, being fitted with Models 1 and 2, respectively. In all other events, the low corona profiles are extrapolated with the corresponding exponential acceleration model.

Here we apply the time-shift technique to seven anisotropic GLEs. For a comparison with CME data, the GLE time is corrected for the difference in Sun-Earth transport time between photons and protons $(8.3$ minute $-\Delta t$ ). Then, the CME profiles given by Equation (3) and Figure 1 are used to convert the GLE time into the CME height, and to plot by this expedient the NM count-rate profile as a function of the CME height.

Figure 2 shows the profiles of three GLEs possessing a steplike onset, hereafter GLEs of morphological type H. From all GLE profiles observed at different stations, we select 1-2 stations where the observed GLE time profile reveals the earliest and steepest rise phase, which implies that the "proton viewing" directions at geomagnetic locations of those stations are close to the axis of the solar proton flux. When plotted as a
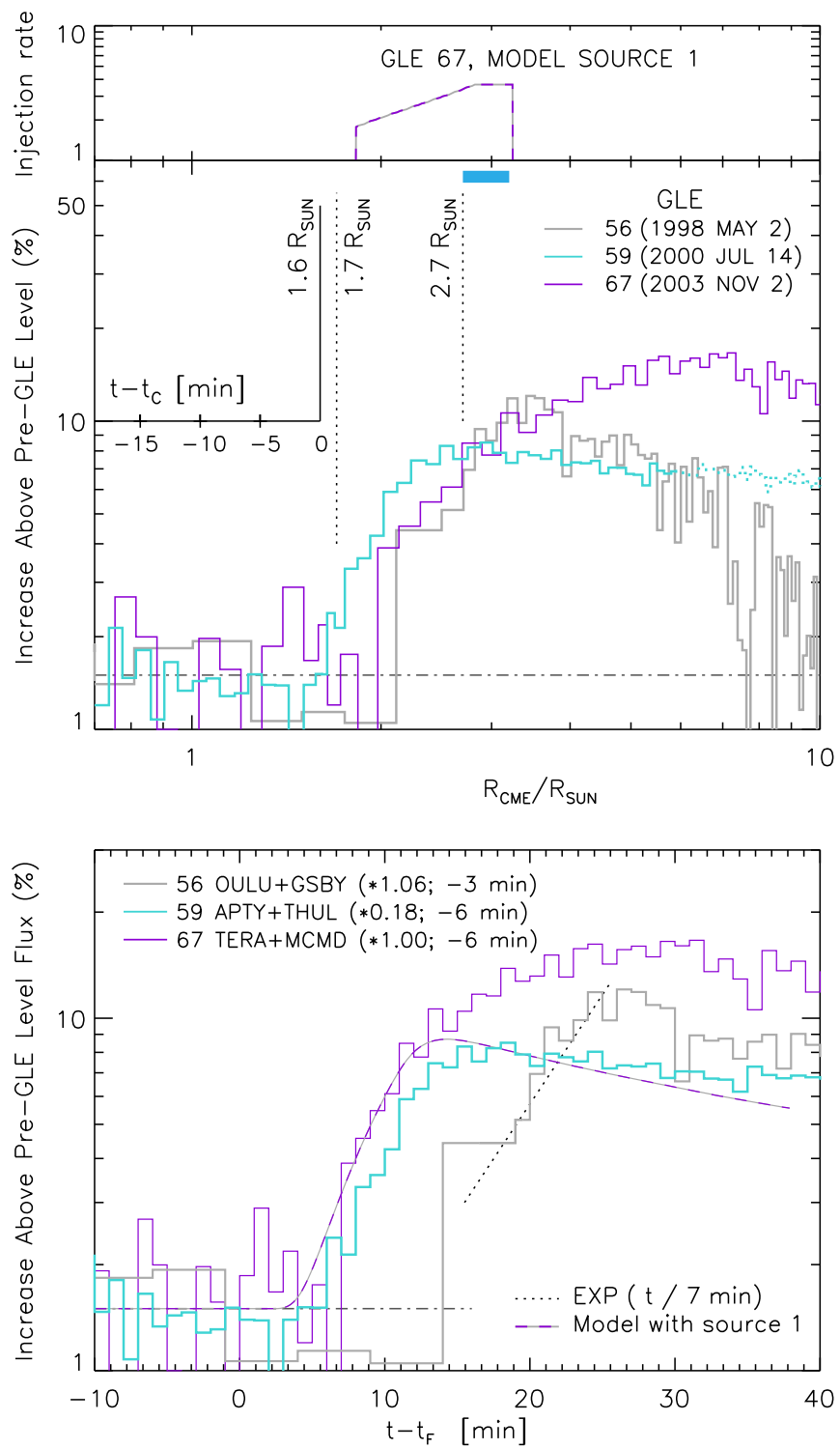

Figure 2. NM count-rate profiles vs. time (lowermost panel) and vs. CME height (middle panel) for three GLEs of type H. For each event, we show the average profile of a pair of "best-oriented" stations: Oulu and Goose Bay for GLE 56, Apatity and Thule for GLE 59, and Terre Adelie and McMurdo for GLE 67, with $98.5 \%$ of pre-GLE level subtracted. The GLE magnitudes are normalized to one and the same value at the height interval indicated with a blue bar at the top of the middle panel (the normalization factors are given in the lower panel along with the time-shift values). The uppermost panel additionally shows the first of two solar sources of protons that were deduced for GLE 67 with a full-scale modeling (Kocharov et al. 2017). The model GLE profile produced by that source is also shown in the lowermost panel. Note: in the middle panel, the count-rate profiles prior the CME launch are plotted as a function of re-scaled time (the secondary axis $t-t_{\mathrm{C}}$; the time scaling factors, $\rho$, are given in the inserts of Figure 1).

function of CME height, all three GLE profiles rise fast between $1.7 R_{\odot}$ and $2.7 R_{\odot}$. Hence, proton emission of type $\mathrm{H}$ appears concurrently with the CME-nose transit from $\approx 2 R_{\odot}$ to $\approx 3 R_{\odot}$, irrespective of the CME speed. Solar electromagnetic emissions associated with this type of GLE were described in detail in our previous paper and will be briefly listed in Section 3.

Shortly after the initial, steep rise, GLEs 56 and 59 start to decline, while GLE 67 slowly rises until $\sim 7 R_{\odot}$. The latter is a signature of the second, less intensive but more prolonged 

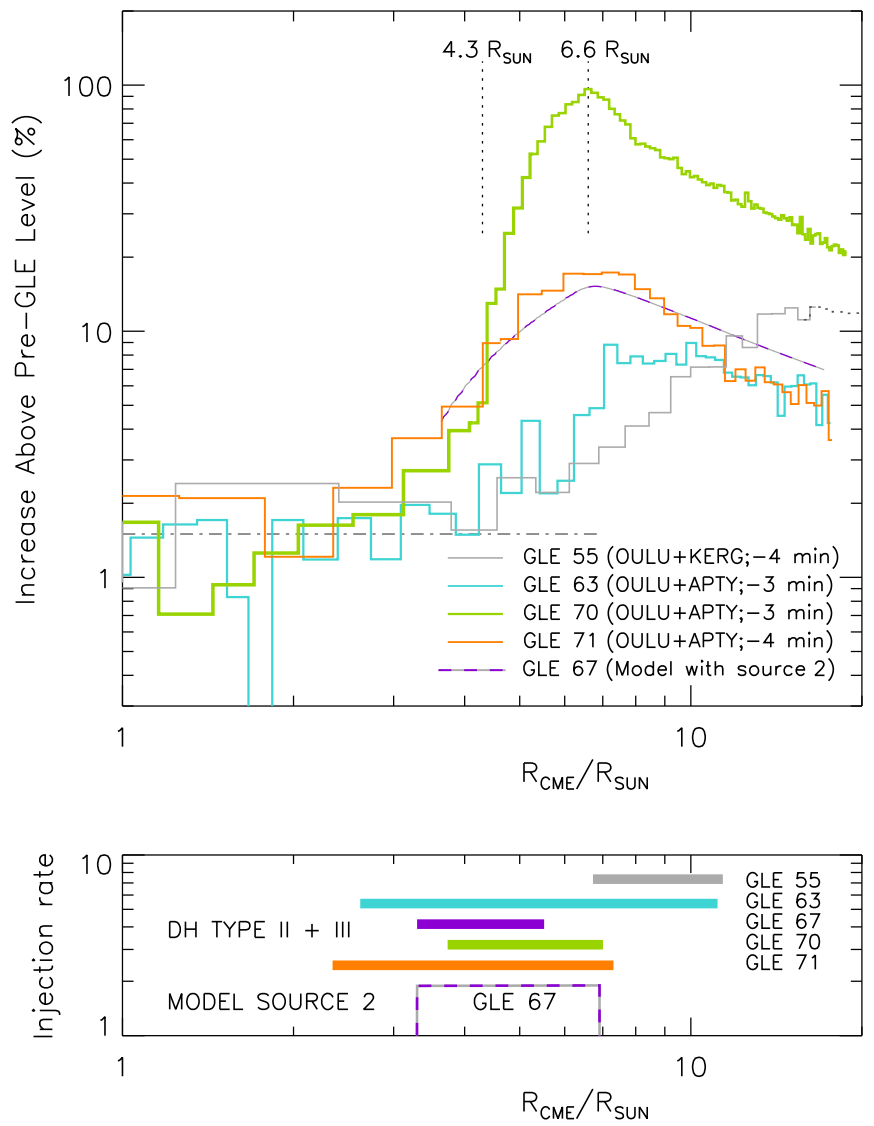

Figure 3. Neutron monitor count-rates vs. CME height for GLEs of type J (upper panel; similar to the middle panel of Figure 2 but not re-normalized) and timing of the associated decametric-hectometric radio emission that includes enhanced type II emission and a concurrent series of low-frequency type III bursts (bars in the lower panel; data of WAVES instruments on board the Wind and Solar Terrestrial Relations Observatory (STEREO) spacecraft). The lower panel also shows the second solar proton source of the GLE 67 model by Kocharov et al. (2017). The model count-rate profile produced by that source is shown in the upper panel.

proton source operating at the Sun (Source 2 in the model by Kocharov et al. 2017, while their Source 1 is responsible for the initial rise between $2 R_{\odot}$ and $3 R_{\odot}$ ). The two model sources of GLE 67 and the corresponding NM profiles are also shown in Figures 2 and 3.

Not all GLEs, however, rise abruptly when a CME expands between $\sim 2 R_{\odot}$ and $3 R_{\odot}$. Figure 3 shows patterns of another kind, in which GLEs gradually rise at $R>3 R_{\odot}$, reach maximum intensity within $10 R_{\odot}$, and then decay, hereafter GLEs of type J. This type of GLE is well exemplified by GLE 70, 2006 December 13. It rises in two stages: (i) a gradual rise occurs between $3 R_{\odot}$ and $4.3 R_{\odot}$, followed by (ii) a fast rise that reaches the maximum magnitude at the CME height of $6.6 R_{\odot}$ and declines shortly after that.

In GLEs 59 and 55, a late part of the maximum phase was not strongly anisotropic (plotted with dotted line in corresponding profiles on Figures 2 and 3). Those dotted parts of the time-shifted profiles may be very different from the source profiles at the Sun because of a strong "blurring" effect of the interplanetary scattering, and could not serve as a proxy of the corresponding solar source.

The decametric-hectometric radio data from the WAVES receivers on the Wind spacecraft (Bougeret et al. 1995) and data from receivers on two spacecraft of the STEREO mission

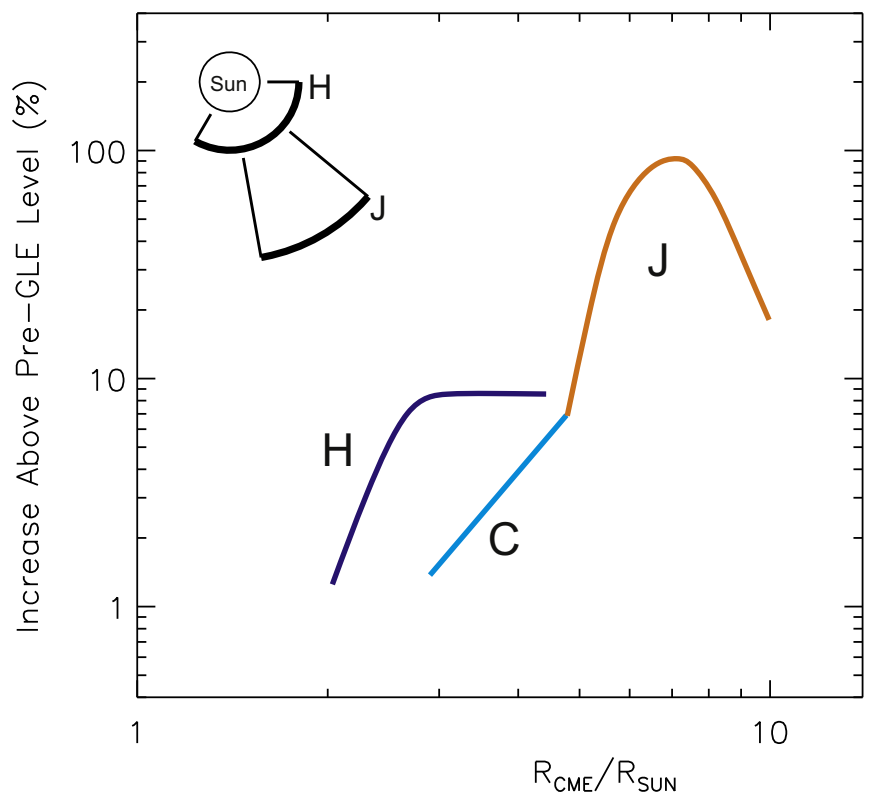

Figure 4. Illustration of GLE profiles of different types as a function of the heliocentric distance of the CME leading edge. GLEs of type $\mathrm{H}$ always rise between $2 R_{\odot}$ and $3 R_{\odot}$. The location of the type $\mathrm{J}$ peak varies between $6 R_{\odot}$ and $10 R_{\odot}$. Emission of type $\mathrm{C}$ rises gradually, so that its visible onset strongly depends on the signal-to-background ratio. The insert qualitatively illustrates the spatial organization of two types of high-energy proton sources. The wide longitudinal spread of the type $\mathrm{H}$ component symbolizes that such protons may be emitted at locations far from the eruption center, while the type $\mathrm{J}$ emission originates from the eruption core.

(Kaiser 2005) indicate that the rise phases of type J GLEs coincide with enhancements of the CME-driven decametrichectometric type II radio burst and the latest, low-frequency type III bursts (Figure 3; exemplified for GLE 67 by Figure 8 of Kocharov et al. 2017, at 17:30 UT-17:50 UT, 2003 November 2).

\section{Discussion}

We have compared GLE profiles with profiles of CMEs observed with $\mathrm{SOHO} / \mathrm{LASCO}$. Since the $\mathrm{SOHO}$ launch in 1995 December, a total 14 GLEs were recorded that are sufficiently strong for such analysis, $\gtrsim 10 \%$ over the galactic background. We report here on half of those events (Table 1). Figure 4 illustrates two typical profiles of the considered GLEs, type $\mathrm{H}$ and type J. In GLE 70 (2006 December 13), the main pulse was preceded by a gradual rise phase (Figure 3), labeled $\mathrm{C}$ in the sketch. Also, observed in GLE 55 (1997 November 6) is an additional emission of relativistic protons when the CMEnose was beyond $10 R_{\odot}$ (Figure 3 ; not sketched in Figure 4 ).

The polar diagram in the insert illustrates the difference in the CME height at the time of the solar proton release in GLEs of type $\mathrm{H}$ and type $\mathrm{J}$. It also makes a reservation for possible difference in the angular spread of the two types of high-energy proton emissions, with no attempt to estimate its exact angular size and possible structure. Both GLEs of pure type $\mathrm{H}$ are observed at nominal magnetic connection, far from the flare location on the solar disk (Table 1). For this reason, we have reserved a wide emission cone for type $\mathrm{H}$ emission (to be verified in the future with a larger statistical sample or stereoscopic observations).

Interplanetary transport modeling indicates that at the proton mean free path $\gtrsim 1 \mathrm{au}$, a prolonged rise, and maximum phase of 
GLE ( $>5$ minute from onset to beginning of decay) implies a prolonged injection of protons from the Sun. This is illustrated by modeling the $\mathrm{J}$ component of GLE 67 (Figure 3). The dashed line in the lower panel shows the proton source (model source 2). A similar curve in the upper panel shows the timeshifted 1 au profile obtained with modeling (for the model description, see Kocharov et al. 2017). While the time shifting of the 1 au profile does not allow the obtaining of an emission profile, the event onset and the beginning of its decay phase can bracket the main emission period.

High-energy proton emission of type $\mathrm{H}$ always appears during the CME-nose transit between $2 R_{\odot}$ and $3 R_{\odot}$. It is associated with a lateral expansion of CME, the extreme ultraviolet (EUV) dimming of extended coronal regions, and low-frequency type III radio bursts whose starting frequencies are lower than the frequencies of previous, flare-associated type IIIs (Kocharov et al. 2017). These observations support the idea that protons of the type H GLEs escape from the Sun when the expanding CME opens the large-scale magnetic loops, where the particles, perhaps accelerated by CME itself, could be released.

The type $\mathbf{J}$ emission coincides with the decametrichectometric type II burst and concurrent bursts of type III (Figure 3). This means that the proton emission is caused by the CME interaction with a previous structure like a coronal streamer, including episodes of magnetic reconnection with open magnetic field lines along which particles can escape to $1 \mathrm{au}$.

Type J GLE of 2006 December 13 (GLE 70) was observed not only by the ground-based NMs but also in space-in the high-energy range, by the Payload for Antimatter Matter Exploration and Light-nuclei Astrophysics (PAMELA) particle instrument (Adriani et al. 2011) and in a lower energy range, by the Energetic and Relativistic Nuclei and Electron (ERNE) instrument on board SOHO (Kocharov et al. 2015). The GLE 70 profile comprises an early, gradual rise phase between $3 R_{\odot}$ and $4.3 R_{\odot}$ (phase $\mathrm{C}$ ), and a steep, major peak at around $6.6 R_{\odot}$ (phase J; Figures 3 and 4). A comparison with $S O H O / E R N E$ data indicates that phase $\mathbf{J}$ starts with injection of helium-rich composition in the energy range $38-87 \mathrm{MeV} \mathrm{nucl}^{-1}$, while phase $\mathrm{C}$ was poor in helium, $\mathrm{He} / p \sim 0.01$ (Figure 4 of Kocharov et al. 2015). In the late phase of the event, a heliumrich composition with $\mathrm{He} / p \approx 0.1$ was observed by PAMELA in the rigidity range 1-3 GV (Figure 7 of Adriani et al. 2011). For these reasons, we think that the particle emission of phase $\mathrm{C}$ and emission of phase $\mathrm{J}$ correspond to two different particle populations originating from two different sources at/near the Sun, while the entire GLE is dominated by emission $\mathrm{J}$.

A helium-rich composition was inferred from the flare $\gamma$-ray observations to exist at the flare site (Mandzhavidze et al. 1999). Helium-rich plasmas are also observed in magnetic clouds arriving at $1 \mathrm{au}$, with $\mathrm{He} / \mathrm{H}>0.08$ (e.g., Lynch et al. 2003). In contrast, helium-poor plasmas are typically met in the high corona and solar wind. Because of the high helium abundance observed in GLE 70, the type $\mathrm{J}$ component of that event could originate from the flaring active region, even though the particle injection into the space is delayed with respect to the flare pulse. Note that in other SEP events, there were similar observations of a delayed helium-rich emission in the deka-MeV nucl ${ }^{-1}$ range, being similarly associated with concurrent decametric-hectometric type II and type III radio bursts (Kocharov et al. 2010).

If the flare-accelerated ions are released into the interplanetary space after a confinement in some magnetic trap, their history should be studied using their secondary neutral emissions like $\gamma$-rays and neutrons. A comprehensive analysis of the secondaries is available for the historic GLE 48 of 1990 May 24 (see in particular Kocharov et al. 1994, 1996a, 1996b). There were no direct observations of associated CME, but a global wave on the solar surface was observed, with a Moreton wave of speed 1500-2600 $\mathrm{km} \mathrm{s}^{-1}$ (Liu et al. 2013). Referring to other events, CME speed may be 1.3-2 times the speed of a Moreton wave on the Sun (e.g., Pohjolainen et al. 2001). When we vary the CME speed estimate from $V_{a}=2500$ to $3500 \mathrm{~km} \mathrm{~s}^{-1}$, the GLE 48 profile as a function of $\mathrm{CME}$ height varies from a profile like that of GLE 67 to a profile that is similar to that of GLE 70; thus, GLE 48 falls into category $\mathrm{H}+\mathrm{J}$ or $\mathrm{J}$. By comparing the energy spectrum of high-energy protons observed at 1 au and the inferred spectrum of protons interacting at the Sun, it was speculated that the flare-accelerated particles were trapped in high coronal loops, where from they were later released after the magnetic field opening (Kocharov et al. 1996a, 1996b).

In general, not all GLEs fall into the two categories considered here, $\mathrm{H}$ and J. For instance, the 2005 January 20 event (GLE 69) starts with an extremely impulsive GLE preceding the low-frequency type II radio burst (Masson et al. 2009). It belongs to a distinct class of impulsive GLEs (McCracken et al. 2012). On the other hand, our GLE 55 (Figure 3) rises once again late in the event, when the CME is above $10 R_{\odot}$ and all type III radio bursts have ceased. A very prolonged emission of relativistic protons has been recently observed in GLE 72, on 2017 September 10. We intend to address those distinct types of GLEs in another work.

\section{Conclusion}

We have considered profiles of seven GLEs depending on the CME expansion phase and find that five of them fall into two clearly distinct categories, type $\mathrm{H}$ and type $\mathrm{J}$, and two events are hybrid. Both types of emissions are concurrent with late, lowfrequency type III radio bursts, indicative of the magnetic field opening for the particle escape. Type $\mathrm{H}$ emission rises stepwise during the CME transit from $2 R_{\odot}$ to $3 R_{\odot}$, irrespective of the CME speed. A straightforward interpretation is that at this stage of expansion, the CME opens the overlying magnetic loops and allows the accelerated protons to escape. Type $\mathrm{J}$ emission peaks much later, when the $\mathrm{CME}$ expands to $6-10 R_{\odot}$. It is concurrent with decametric-hectometric type II brightening and could be released at the CME interaction with a streamer-like structure, while the particle abundance, still known only in one event, is rich in helium, which may indicate that the high-energy ions initially originate from the flare region. The contribution of a gradual component that would be expected from the CME-bow-shock acceleration in unstructured solar wind seems small in the type $\mathrm{H}$ and type $\mathrm{J}$ events considered here.

This research was supported by the Academy of Finland through projects 304435 (CRIPA-X), 260596, and 267186. 


\section{ORCID iDs}

Leon Kocharov (i) https://orcid.org/0000-0002-2077-5618

Haimin Wang (i) https://orcid.org/0000-0002-5233-565X

Ilya Usoskin (i) https://orcid.org/0000-0001-8227-9081

\section{References}

Adriani, O., Barbarino, G. C., \& Bazilevskaya, G. A. 2011, ApJ, 742, 102 Aschwanden, M. J. 2012, SSRv, 171, 3

Bougeret, J.-L., Kaiser, M. L., Kellogg, P. J., et al. 1995, SSRv, 71, 231

Brueckner, G. E., Howard, R. A., Koomen, M. J., et al. 1995, SoPh, 162, 357

Cliver, E. W. 2016, ApJ, 832, 128

Gopalswamy, N., Xie, H., Akiyama, S., et al. 2013, ApJL, 765, L30

Gopalswamy, N., Xie, H., Yashiro, S., et al. 2012, SSRv, 171, 23

Kahler, S. 1994, ApJ, 428, 837

Kaiser, M. L. 2005, AdSpR, 36, 1483

Kocharov, L., Klassen, A., Valtonen, E., Usoskin, I., \& Ryan, J. M. 2015, ApJL, 811, L9

Kocharov, L., Kovaltsov, G., Torsti, J., et al. 1996a, in AIP Conf. Proc. 374, High Energy Solar Physics, ed. R. Ramaty, N. Mandzhavidze, \& X.-M. Hua (Woodbury), 246

Kocharov, L., Pohjolainen, S., Mishev, A., et al. 2017, ApJ, 839, 79
Kocharov, L., Reiner, M. J., Klassen, A., Thopmpson, B. J., \& Valtonen, E. 2010, ApJ, 725, 2262

Kocharov, L., Saloniemi, O., Torsti, J., et al. 2007, ApJ, 659, 780

Kocharov, L., Torsti, J., Vainio, R., Kovaltsov, G. A., \& Usoskin, I. G. 1996b, SoPh, 169, 181

Kocharov, L. G., Lee, J. W., Zirin, H., et al. 1994, SoPh, 155, 149

Liu, R., Liu, C., Xu, Y., et al. 2013, ApJ, 773, 166

Lovell, J. L., Dudling, M. L., Humble, J. E., Shear, M. A., \& Flükiger, E. O. 2002, AdSpR, 30, 1045

Lynch, B. J., Zurbuchen, T. H., Fisk, L. A., \& Antiochos, S. K. 2003, JGR, 108,1239

Mandzhavidze, N., Ramaty, R., \& Kozlovsky, B. 1999, ApJ, 518, 918

Masson, S., Klein, K.-L., Bütikofer, R., et al. 2009, SoPh, 257, 305

McCracken, K. G., Moraal, H., \& Shea, M. A. 2012, ApJ, 761, 101

Mishev, A. L., Kocharov, L. G., \& Usoskin, I. G. 2014, JGRA, 119, 670

Pohjolainen, S., Maia, D., Pick, M., et al. 2001, ApJ, 556, 421

Reames, D. V. 1999, SSRv, 90, 413

Shea, M. A., \& Smart, D. F. 2012, SSRv, 171, 161

Vashenyuk, E. V., Balabin, B. B., \& Gvozdevsky, B. B. 2003, in Proc. 28th Int. Cosmic Ray Conf., ed. T. Kajita et al. (Tsukuba: Universal Academy Press), 3401

Vashenyuk, E. V., Balabin, B. B., Gvozdevsky, B. B., \& Shchur, L. I. 2008, Ge\&Ae, 48, 149

Yashiro, S., Gopalswamy, N., Michalek, G., et al. 2004, JGR, 109, A07105 